\title{
Chemically reactive turbulent vortex rings
}

\author{
H. Johari \\ Department of Mechanical Engineering, Worcester Polytechnic Institute, Worcester, Massachusetts 01609
}

(Received 2 November 1993; accepted 22 June 1995)

\begin{abstract}
Employing an aqueous acid-base reaction, the minimum mixing rate of turbulent vortex rings was investigated in a water tank. Vortex rings were generated by a simple apparatus with a cylindrical geometry. The released fluid surrounding the vortex core mixed very rapidly when compared with the fluid in the toroidal core. Moreover, the fluid within the core did not mix uniformly in the azimuthal direction. The normalized distance a vortex ring must travel, in order to completely mix with the ambient fluid to a specific volumetric ratio, depends on the aspect ratio of the generating cylinder. Scaling arguments are presented that relate the above distance to the spreading rate and the generating apparatus parameters. Due to the very small net entrainment rate of vortex rings, the detrainment of core material cannot be ignored when the mixing rate of the core is considered. (C) 1995 American Institute of Physics.
\end{abstract}

\section{INTRODUCTION}

A vortex ring is created when a fluid parcel is impulsively discharged from a circular opening into a quiescent environment. Vortex rings have been studied for more than a century primarily due to their ability to transport mass and momentum for a relatively long distance with little mixing. The application of vortex rings to industrial practices has also been discussed in the literature. For example, Turner ${ }^{1}$ studied the feasibility of transporting pollutants to high altitudes via buoyant vortex rings. It has also been suggested that vortex rings can be employed to improve the mixing of fuel injected into a high-speed cross-flow. ${ }^{2}$ Knowledge of the mixing rate in vortex rings is crucial in the above examples, as well as other applications.

There exists a large body of work on the formation, structure, and stability of laminar vortex rings. ${ }^{3-5}$ At large Reynolds numbers, vortex rings are either turbulent at birth or become turbulent after going through ' a transition phase. ${ }^{6}$ A major difference between laminar and turbulent vortex rings is that turbulent rings leave mixed fluid in their wake. A concise review of vortex rings was recently carried out by Shariff and Leonard. ${ }^{7}$

Maxworthy ${ }^{8}$ conducted flow visualization studies of turbulent vortex rings and described the flow in terms of a core of very fine-scale turbulence surrounded by a co-moving "bubble." Ambient fluid was observed to get entrained into the bubble; a small portion was retained while the rest was ejected into the wake after mixing with the vortex fluid. These observations were later corroborated by Glezer and Coles. ${ }^{9}$ Maxworthy also presented arguments for the celerity of the vortex bubble, which are in contrast to the classical self-similar scaling (based on the conservation of impulse assumption). Johnson ${ }^{10}$ used an alternative power law to describe the motion of turbulent vortex rings.

Even the available data on the spreading rate of conically growing turbulent vortex rings have a wide range with the smallest reported value ${ }^{11}$ of $0.5 \times 10^{-3}$ and the largest value $^{8}$ of $12 \times 10^{-3}$. This is in contrast to the consistent measurements of spreading rates of other free shear flows. The spreading, rate of vortex rings, generally much smaller than other free shear flows, is dependent on the generating apparatus geometry as well as the Reynolds number. ${ }^{11}$ It has also been reported that at far downstream locations, a turbulent vortex ring may undergo a transformation that changes the spreading rate. ${ }^{11,12}$ At present there does not exist a method of predicting the spreading rate of a turbulent vortex ring, even if its generating geometry and initial Reynolds number are known.

In the context of mixing, the entrainment and detrainment rates of the bubble, as well as the mixing rate of the vortex core, are important. Since vorticity is concentrated in a small area associated with the vortex core, the core region has the slowest mixing rate. Based on the appropriately averaged velocity measurements, Glezer and $\mathrm{Coles}^{9}$ were able to determine the net entrainment rate of the bubble. The entrainment processes in vortex rings were qualitatively described by Auerbach ${ }^{13}$ on the basis of flow visualization studies. Detailed planar measurements of a passive scalar (dye concentration) field within the initial phases of a laminar vortex ring enabled Southerland et al. ${ }^{14}$ to calculate the local (fine-scale) mixing rate.

The present study is concerned with the molecular scale mixing rate of turbulent vortex rings, as revealed by an isothermal chemical reaction (acid-base neutralization) in the large Schmidt number regime. Of particular interest is the dependence of the mixing on the vortex generator geometry and Reynolds number. Scaling arguments that provide the distance within which the vortex core mixes to a certain volumetric ratio are presented in Sec. III. Experiments were performed to measure the core mixing rate and to verify the validity of the proposed scaling.

\section{APPARATUS}

The experiments were conducted in a transparent acrylic water tank having dimensions $1.2 \times 1.2 \times 1.5 \mathrm{~m}$ deep. The vortex generating apparatus consisted of an acrylic cylinder sealed on the top by a valve and on the bottom by a thin sliding stainless steel plate. The cylinder was positioned on top of a $30 \mathrm{~cm}$ square platform with the sliding plate resting on the platform. The sliding plate is needed to separate the 

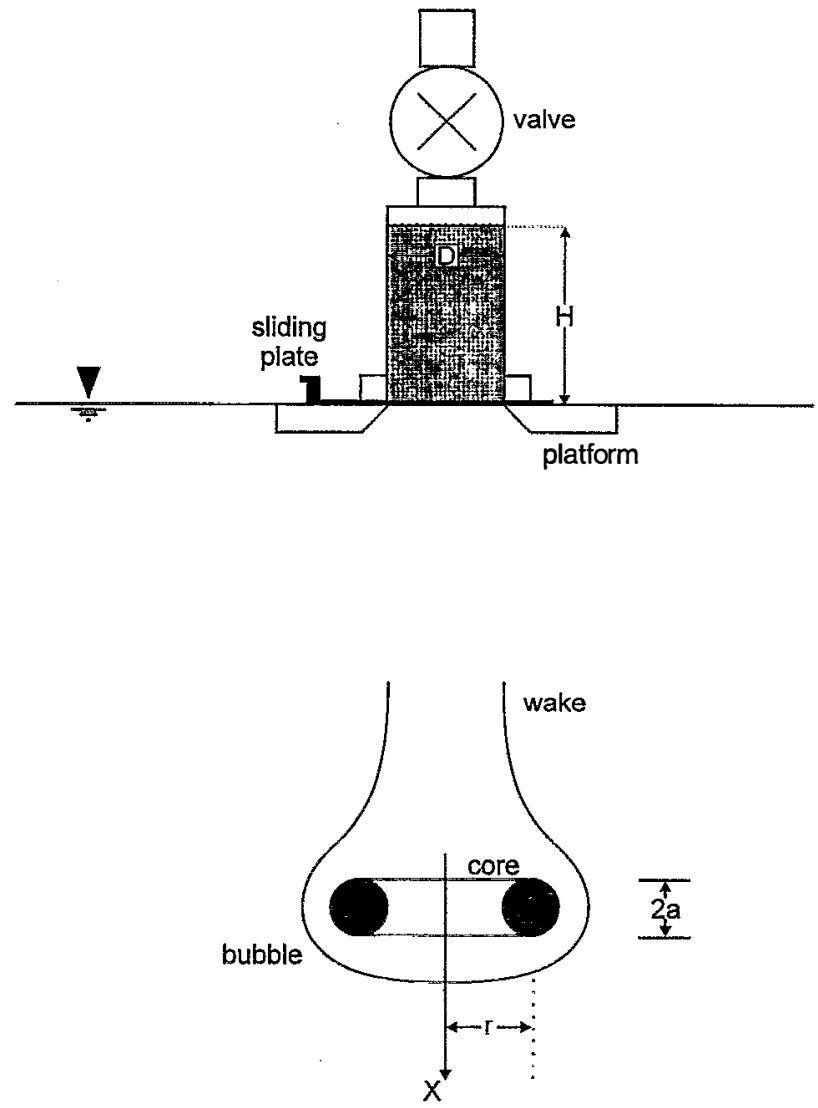

FIG. 1. Schematic of vortex ring generator and vortex core (not to scale).

chemically reactive reservoir and ambient fluids. The platform had a circular opening underneath the cylinder. The vortex generator assembly was placed above the water tank such that the sliding plate was at the free surface. Three different cylinders with $3.8,7.5$, and $10.1 \mathrm{~cm}$ diam could be placed in the apparatus. A schematic drawing of the vortex generator is shown in Fig. 1.

The released fluid was fed into the cylinder with the valve in the open position. The valve was closed subsequently and the fluid was allowed to become quiescent. The sliding plate was pulled back smoothly, leaving the released fluid in contact with the tank fluid. Although care was taken to minimally disturb the interface between the two fluids, a laminar wake appeared due to the motion of the plate. A crude estimate of $3 \mathrm{~cm} / \mathrm{s}$ for the plate velocity results in a maximum boundary layer displacement thickness of $0.32 \mathrm{~cm}$ for the largest cylinder. The width of the wake, which would be twice this value, is still small in comparison with the cylinder dimensions. In any run, time was allowed for the motions to subside prior to the release. The fluid remained in the cylinder since the valve on top of the cylinder was closed. No mean flow can be established with the valve in the closed position. The flow was initiated when the valve was rapidly opened. Gravity forced the discharge of vortex fluid into the water tank, thus creating a vortex ring. Since it was not necessary to fill the entire cylinder with released fluid, various height $H$ to diameter $D$ ratios could be achieved in this setup. All three cylinders were $15.2 \mathrm{~cm}$ deep, resulting in maximum $H / D$ ratios of $1.5,2$, and 4 for 10.1 , 7.5 , and $3.8 \mathrm{~cm}$ diam cylinders, respectively.

Classically, vortex rings have been visualized by marking the vortex fluid with an inert dye. Such a visualization provides an integrated external view appropriate for measurement of global properties. Recently, planar laser-induced fluorescence techniques have been employed for visualization of the intimate details of vortex cores in selected planes. ${ }^{6,14}$ In the present experiments, an aqueous acid-base neutralization reaction along with a $p H$ indicator (phenolphthalein) was employed. The tank fluid contained a $10^{-2}$ molar acidic $\left(\mathrm{H}_{2} \mathrm{SO}_{4}\right)$ solution. Phenolphthalein, in ethanol solution, was mixed with the released fluid, which was a weak alkaline $(\mathrm{NaOH})$ solution. The indicator had a purplish color in the alkaline solution. Upon mixing with the ambient fluid to a prescribed volumetric ratio $\phi$, the indicator was rendered colorless. Here $\phi$ is the acid-base stoichiometric ratio, the ratio of volume (mass) of acidic solution required to neutralize a unit volume (mass) of the alkaline solution. In this manner the location of the last parcel to molecularly mix with the ambient fluid could be determined. The distance from the generator exit to this location was termed "reaction length." The reaction length is a measure of the minimum molecular scale mixing in the vortex ring, since the time scale of the acid-base reaction is short compared with the fluid mechanical time scales. The density difference between the released and tank fluid was kept below $0.1 \%$, thereby minimizing any buoyancy related effects.

Figure 2 compares two turbulent vortex rings, one marked by an inert dye while the other containing the $p H$ indicator. Both were generated from the same $(10.1 \mathrm{~cm}$ diam) cylinder. The "bubble" fluid, as well as the wake, are clearly visible in the inert dye vortex. However, the chemically reactive vortex ring reveals only the toroidal core, since the rest of the released fluid has mixed with the ambient fluid and has been neutralized (except for a small portion near the vortex generator). Eventually, the core will also mix and discolor when the vortex ring reaches its reaction length. This technique has previously been used to measure the mixing rate in turbulent jets. ${ }^{15,16}$

The weight of the fluid slug above the free surface of the tank provided the necessary impulse for the motion, in the present vortex generator. In order to calculate the impulse $I$, the velocity time history during the discharge is needed. If one neglects the effects of boundary layers, the velocity time history could be found from the unsteady Bernoulli's equation. Neglect of the boundary layer thickness is justifiable, since the resulting area contraction at the end of a run amounts to about 1.5 and $3 \%$ for the large and small cylinders, respectively. Because the bottom of the cylinder is at atmospheric pressure, Bernoulli's equation provides a linear velocity time history, i.e., $U=g t$, where $g$ is the gravitational acceleration. The discharge time $T$ is $(2 H / g)^{1 / 2}$, where $H$ is the initial height of the fluid slug. Since $T$ is an estimate of the actual discharge time, then values of other parameters based on it are estimates as well.

Assuming a uniform velocity and density across the cylinder exit of area $A$, the vortex ring impulse, defined as the momentum associated with the discharge, is as follows: 

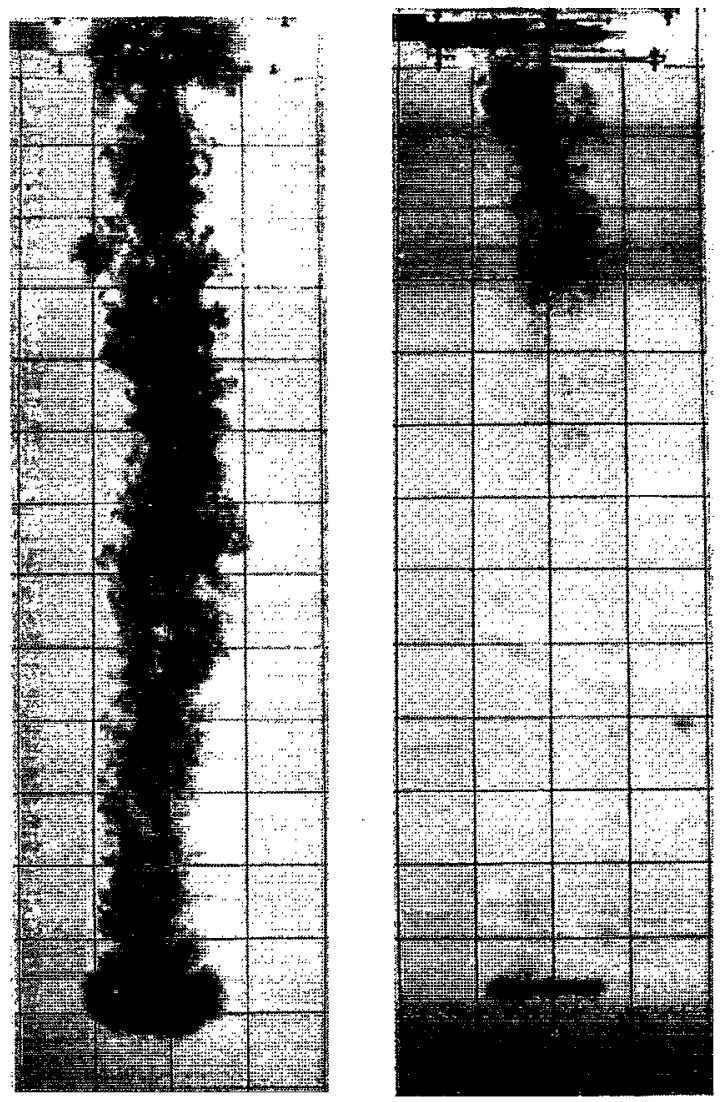

FIG. 2. Photographs of chemically passive (left) and reactive (right) vortex rings, $D=10.1 \mathrm{~cm}, H / D=1.5$, and $\operatorname{Re}=8.8 \times 10^{4}$. The grid lines are $10 \mathrm{~cm}$ apart.

$$
I=\rho A \int_{0}^{T} U^{2}(t) d t=\rho A\left(\frac{1}{3} g^{2} T^{3}\right) .
$$

Based on this expression, the initial circulation $\Gamma_{0}$ is given ${ }^{11}$ by

$$
\Gamma_{0}=\frac{I}{2 \rho A}=\frac{1}{6} g^{2} T^{3}=\frac{\left(2 g H^{3}\right)^{1 / 2}}{3} .
$$

An initial Reynolds number $\mathrm{Re}_{0}$ can be specified by the ratio of the initial circulation over the kinematic viscosity $\nu$ $\left(\operatorname{Re}_{0}=\Gamma_{0} / \nu\right)$. In classifying our results, this initial Reynolds number and the generator aspect ratio $H / D$ will be utilized. It is noteworthy that the initial circulation (or $\mathrm{Re}_{0}$ ) and the discharge time only depend on the fluid slug height in the cylinder and not the cylinder diameter. Therefore, the initial Reynolds number and the aspect ratio could be varied independently by employing the three cylinders.

For the small cylinder with $D=3.8 \mathrm{~cm}, H / D$ was varied in the range of 4 to 1.5 , resulting in initial Reynolds numbers of 8.8 to $2.0 \times 10^{4}$. For the medium cylinder with $D=7.5 \mathrm{~cm}$, $H / D$ values in the range of 2 to 1 were used with accompanying Reynolds numbers in the range of 8.8 to $3.1 \times 10^{4}$. Only $H / D$ values between 1.5 and 1.0 were utilized with the large cylinder $(D=10.1 \mathrm{~cm})$. The Reynolds number range for the latter set was from 8.8 to $4.8 \times 10^{4}$. According to the transition chart provided by Glezer, ${ }^{6}$ all the vortex rings generated by the apparatus in the present range of parameters
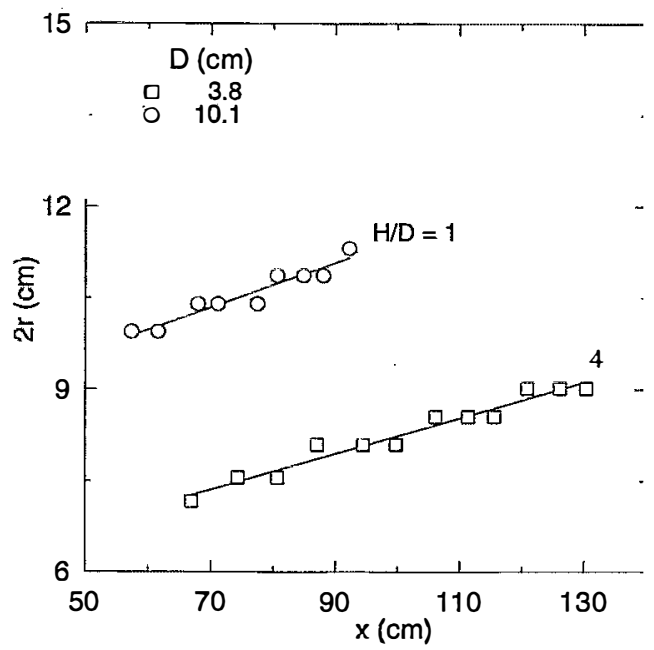

FIG. 3. Vortex ring diameter $2 r$ as a function of axial position $x$, in the similarity stage. The solid lines represent least square lines fitted to each dataset.

should be turbulent at birth. The observed vortex rings were either turbulent at birth or became turbulent within the first few diameters, with the exception of rings created by the small cylinder at lower Reynolds numbers. Vortex rings with $H / D$ values smaller than unity were not attempted in the present study.

Initially, a series of runs were performed in which the ring diameter $(2 r)$ and the axial position of the core were measured as a function of time. The spreading rate $\alpha$, defined as $d r / d x$, can be obtained from a plot of torus diameter against position. Figure 3 shows such a plot for two cases. The data were measured visually from photographs and the uncertainty in torus diameter is about $\pm 5 \%$. The spreading rate was found by fitting the best straight line through the data points. Table I lists the spreading rates for four cases studied in detail, along with the discharge time, $H / D$, and $\mathrm{Re}_{0}$. The spreading rates range from 0.0125 to 0.018 and a mean value of 0.015 will be assumed for further discussions.

There are two important issues related to the data in Fig. 3 that need to be considered. The first one is the zigzag nature of the data where the ring expands suddenly and then remains constant prior to another jump. Even though the growth of vortex rings is not, in general, a smooth function of distance or time, the zigzag behavior in our data is caused by the resolution limitations in the measurements of ring diameter. The uniform magnitude of jumps in the data confirms this explanation.

TABLE I. Spreading rate and generating parameters of vortex rings.

\begin{tabular}{ccccc}
\hline \hline & & & & \multicolumn{1}{c}{} \\
$D(\mathrm{~cm})$ & $H / D$ & $T(\mathrm{~ms})$ & $\operatorname{Re}_{0} \times 10^{-4}$ & $\left(=\frac{d r}{d x}\right)$ \\
3.8 & 4.0 & 176 & 8.8 & 0.014 \\
7.5 & 2.0 & 176 & 8.8 & 0.015 \\
10.1 & 1.5 & 176 & 8.8 & 0.0125 \\
10.1 & 1.0 & 144 & 4.8 & 0.018 \\
\hline
\end{tabular}


The second issue to be addressed is the relatively large growth rates of the vortex rings created by the present generator. The observed growth rates are larger than the measured values of Glezer and Coles $^{9}(H / D=3.42)$ and Sallet and Widmayer ${ }^{12}(H / D<1)$ by a factor of 2 . The lowest reported ${ }^{11}$ spreading rate of 0.0005 was achieved with a generator aspect ratio of 1.51 at Reynolds numbers comparable to the present experiments. Maxworthy's ${ }^{8}$ earlier spreading rate data $\left(11 \times 10^{-3}\right)$ at a Reynolds number around $10^{4}$ are quite close to our data. Maxworthy ${ }^{11}$ also reported relatively larger spreading rates when low Reynolds number turbulent rings were studied. It is hypothesized that our initial Reynolds number calculation, which is based on the "slug" model of ring formation, overestimates the actual $\mathrm{Re}_{0}$ values. Since our $\operatorname{Re}_{0}$ is essentially $\Gamma_{0}$, the actual circulation is thought to be less than that predicted by the impulse of the reservoir fluid. Several factors can contribute to this loss, with the major one being the creation of opposite sign vorticity due to the secondary flow on the generator platform. The generator geometry with the bottom of the platform being at a $45^{\circ}$ angle away from the exit of generator exacerbates this loss. The loss of initial circulation due to the cancellation of core vorticity by the vorticity of opposite sign on the generator walls has been studied in detail by Didden. ${ }^{5}$

An alternative explanation for the large observed spreading rates might be the interference caused by the vorticity left over from the removal of the plate. Even small motions can alter the amplification rate of the instabilities. However, this argument is unlikely to be the prime cause for the large spreading rates, because by the time the vortex ring was released, the motions from the plate removal had essentially subsided. Moreover, since the plate is pulled in one direction, the created vorticity would have one sign all around the cylinder. The core vorticity would be enforced on one side of the cylinder while on the other side, the created vorticity would be of opposite sign to the core vorticity. The result would be vortex rings with consistently uneven growth rates in one particular orientation. Although there were some rings with uneven cores after the formation stage, the majority had relatively uniform cores and more importantly, uniform growth rates. Therefore, the loss of initial circulation appears to be the principal cause of the large observed spreading rates, even though other factors may have contributed.

\section{SCALING ARGUMENTS}

In this section volumetric arguments are derived that relate the reaction length $L$ for a prescribed (volume) mixture ratio $\phi$, with the vortex generator geometry and the ring parameters. The scaling will be limited to turbulent vortex rings at high Reynolds number. Assuming that fluid parcels in the core will be the last ones to mix, the entrainment rate of toroidal ring in the far field becomes the limiting parameter. The volume of a torus with radius $r$ and core radius $a$ is $2 \pi^{2} a^{2} r$. The ring radius increases linearly with axial distance $x$ as follows:

$$
r(x)=r_{0}+\alpha x,
$$

where $r_{0}$ is the extrapolated ring radius at the generator and $\alpha$ is the far field spreading rate. Furthermore, for the ring to be self-similar in the far field, the core radius has to be proportional to the ring radius, $a \sim r$. The ring volume scales with the ring radius to the third power, $V \sim r^{3}$.

A global measure of volumetric entrainment $E$ for vortex rings can be established by the ratio of core volume at any axial distance to the released volume at the generator, $E=V / V_{0}$. Following the Broadwell and Breidenthal ${ }^{17}$ line of reasoning for an entrainment-limited mixing process, $E$ should be proportional to the volumetric mixture ratio $(\phi$ +1 ). The assumption of entrainment-limited mixing is only valid as long as diffusion across small scales occurs faster than entrainment of fresh reactants by the vortex core. ${ }^{17}$ This condition translates to $\operatorname{Re} S c \gg 1$, which appears to hold true in the present experiments (Sc refers to Schmidt number). Therefore, when every fluid parcel in the core is (molecularly) mixed with the ambient fluid to a ratio $\phi$ at axial position $x=L$, the core discolors. In other words, the reaction length $L$ is the axial position where the entire core fluid is mixed with the entrained ambient fluid,

$$
E=\frac{V(x=L)}{V_{0}}=1+\frac{V_{\text {entrained }}}{V_{0}} \sim(1+\phi) .
$$

From here, one can expand the ring volume in terms of the spreading rate $\alpha$ and $L$ as follows:

$$
\frac{r_{0}+\alpha L}{V_{0}^{1 / 3}} \sim(1+\phi)^{1 / 3}
$$

or

$$
L \cong \frac{k(1+\phi)^{1 / 3} V_{0}^{1 / 3}}{\alpha}-\frac{r_{0}}{\alpha},
$$

where $k$ is a proportionality constant. The above expression relates the reaction length with the spreading rate, volumetric mixture fraction, and the released volume. However, there are two concerns about this expression. First, the parameter $r_{0}$ cannot be predicted accurately without knowing the spreading rate and the virtual origin. Since some mixing is expected to occur in the formation stages of the ring, the term containing $r_{0}$ cannot be ignored. An alternative is to assume that $r_{0}$ is equal to the radius of the generating cylinder $D / 2$. Now, the above expression can be rewritten as

$$
\frac{L}{D} \cong\left[C(1+\phi)^{1 / 3}\left(\frac{H}{D}\right)^{1 / 3}-0.5\right] \alpha^{-1},
$$

where $C$ is another proportionality constant and the reaction length, normalized by the cylinder diameter, is a function of the mixture ratio, vortex generator aspect ratio, and the spreading rate. In this expression, all the parameters can be quantified accurately. Equation (6) applies only to generators with cylindrical geometry, whereas the expression in Eq. (5) is a general statement. Even small values of mixture fraction are expected to produce large reaction lengths since $\alpha$ is a small number.

The second concern is in regard to the way volumetric entrainment $E$ was defined. One could perhaps argue that the far field volume of the core should be normalized with the volume of the core after it had achieved a self-similar state. Presently, it is not possible to predict the dimensions of the 
core at the point self-similarity is attained. Furthermore, the dilution of the core material prior to the similarity stage would be difficult to estimate. Therefore, the expression in Eq. (6) will be chosen as a first step toward quantifying the mixing in turbulent vortex rings, with the understanding that this model can be further modified once more knowledge becomes available about the physics of turbulent vortex rings.

Finally, it is worth stating that the Reynolds number does not appear in the above discussions, even though the core material originates from the boundary layers of the vortex generator. The proportionality constant in Eq. (6) may be a weak function of Reynolds number. At high Reynolds numbers, it is expected that global features are independent of viscosity, especially if the flow has achieved self-similarity.

\section{RESULTS}

The results from observations of chemically reactive vortex rings will be presented in this section. Qualitative observations will be discussed first before the presentation of reaction length measurements. Because of the very small growth rate of vortex cores and the limited depth of the water tank, only stoichiometric mixture ratios of less than unity could be utilized, i.e., $\phi<1$. This means that only a small volume of tank fluid was required to render a large volume of released fluid colorless. The majority of runs were performed with a stoichiomeric mixture ratio of $\frac{1}{37}$, although values up to $\frac{1}{5}$ were also used. At these low values of $\phi$, the fluid parcels surrounding the toroidal core discolored within the first few cylinder diameters. Then, the core traveled a certain distance before the core's color also faded. This behavior was expected since the bubble processes a large volume of ambient fluid and the core mixes very slowly.

The most surprising observation was that the core fluid did not mix uniformly, i.e., certain portions of the core neutralized prior to others. Figure 4 depicts a vortex ring in which the front half has mixed with the tank fluid to a volume fraction $\frac{1}{37}$, while the rest of the ring has not. The fluid within the cylinder was a homogeneous solution prior to release. The wake, partially visible in the photograph, was in the process of mixing and discoloration. The fluid left behind near the vortex generator did not have enough turbulent kinetic energy to mix prior to laminarization. This is evident in Fig. 4 and in the chemically reactive vortex ring of Fig. 2 . The location of the first (or last) parcels within the vortex ring to $\mathrm{mix}$ to the required mixture ratio for discoloration varied for different runs. A priori determination of the first parcel to discolor was not possible. Moreover, the position of the first neutralized parcels did not correspond with the direction in which the plate was removed before the beginning of each experiment. For example, the front portion of the ring in Fig. 4 was neutralized first, despite the right to left motion of the plate. In other cases, the back or the sides of the ring discolored first. At present, no satisfactory explanation for the asymmetric mixing around the ring is available, although the generator design (including the plate) may be a factor. Any generator design: without a dividing plate may not be practical, since premature mixing of reactants can occur at the exit plane preceding the release.

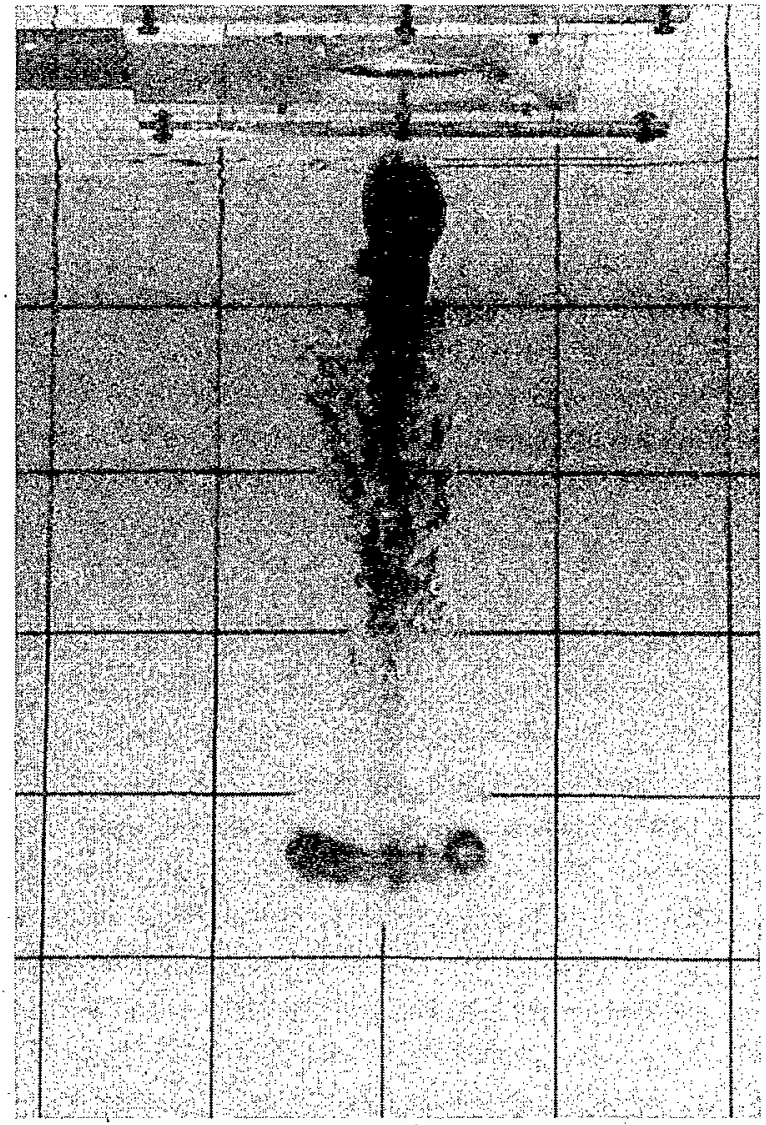

FIG. 4. Asymmetric mixing of a vortex core; $D=7.5 \mathrm{~cm}, H / D=2$; and $\phi=\frac{1}{37}$.

In order to investigate the effect of initial Reynolds number $\left(\Gamma_{0} / \nu\right)$ on the reaction length, a series of runs were performed in which the stoichiometric mixture ratio $\phi$ and the generator aspect ratio $H / D$ were held constant. We recall that the reaction length is the distance from the generator exit beyond which all the released fluid has mixed and reacted with the tank fluid to a volumetric ratio $\phi$. Figure 5 is a plot of reaction length, normalized by $D$, versus the initial Reynolds number for $H / D$ values of $2.0,1.5$, and 1.0. Each data point in the plot is an average of at least five runs and the vertical bars associated with each data point indicate the maximum and minimum observed reaction lengths. An explanation for the existence of $\mathrm{Re}_{0}$ effects is that the formation process of rings is Reynolds number dependent. ${ }^{11}$ Therefore; the initial ring dynamics and possibly the spreading rate are $\mathrm{Re}_{0}$ dependent.

There appears to be at least a weak Reynolds number dependence for all three aspect ratios. The Reynolds number seems to affect the $H / D=2.0$ and 1.0 cases minimally. The difference between the two data points in each set are within our measurement uncertainty of $\pm 5 \%$. The strongest Reynolds number effect was observed when $H / D=1.5$. The normalized reaction length decreased by $15 \%$ when $\mathrm{Re}_{0}$ was increased by a factor of 4 . The variance between the reaction length of 10.1 and $7.5 \mathrm{~cm}$ cylinders is about $4 \%$ for $H / D$ of 1.5 , which is again within our uncertainty band. The larger deviation of about $10 \%$ between the $D=3.8 \mathrm{~cm}$ reaction 


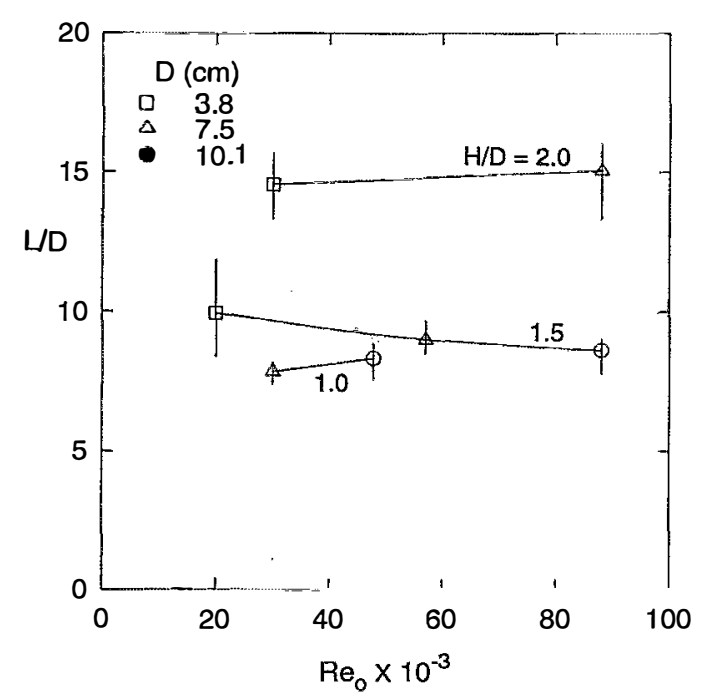

FIG. 5. Reynolds number dependence of normalized reaction length for vortex generator aspect ratios of 2.0,1.5, and 1.0. The vertical bars around each data point indicate the maximum and minimum observed reaction lengths. For each $H / D$, the data points, which correspond to the average value of the measured reaction lengths, are connected by solid lines.

length and the other two data points at this $H / D$ is thought to be due to the likely laminarization of vortex rings from the smallest cylinder. The vortex rings created by the $3.8 \mathrm{~cm}$ cylinder having $H / D$ values of 1.0 and 1.5 showed signs of laminarization at a certain distance from the generator, as noted in Sec. II. The largest scatter, as denoted by the length of vertical bars in Fig. 5, is also associated with the data point resulting from the small cylinder and $H / D=1.5$. Barring the lowest $\mathrm{Re}_{0}$ runs, the influence of the Reynolds number appears to be within our measurement uncertainty for the range of parameters in the experiments.

Another notable aspect of the plot in Fig. 5 is that the reaction length appears to be decreasing with $\mathrm{Re}_{0}$ for the $H / D=1.5$ while increasing for $H / D$ values of 1.0 and 2.0. Although the pattern in the former case appears to be more logical due to the greater mixing at higher $\mathrm{Re}$, the pattern in the latter cases has also been observed in gaseous shear layers, where the normalized product formation decreases with increasing Reynolds number. Both increasing and decreasing reaction lengths, with respect to $\mathrm{Re}$, have been observed in steady jets, before the reaction length becomes independent of Reynolds number. ${ }^{16}$

The next step is to check whether the relationship in Eq. (6) is valid. Since the stoichiometric mixture ratio could not be varied extensively, the value of $\frac{1}{37}$ was utilized in a number of runs. The aspect ratio of the vortex generator was varied from 1 to 4 among the three cylinders. Normalized reaction length is plotted against $(H / D)^{1 / 3}$ in Fig. 6. The initial Reynolds number for all the data is greater than $3 \times 10^{4}$. Reaction lengths exceeding $85 \%$ of the available tank depth were discarded in order to exclude the data influenced by vortex ring interactions with the tank bottom. A least-squares line was fitted to the data, and it appears that the reaction length is linearly proportional to $(H / D)^{1 / 3}$. The line has a slope of 24.3, which translates to a value of 0.36 for constant $C$ in Eq. (6), assuming an average value of

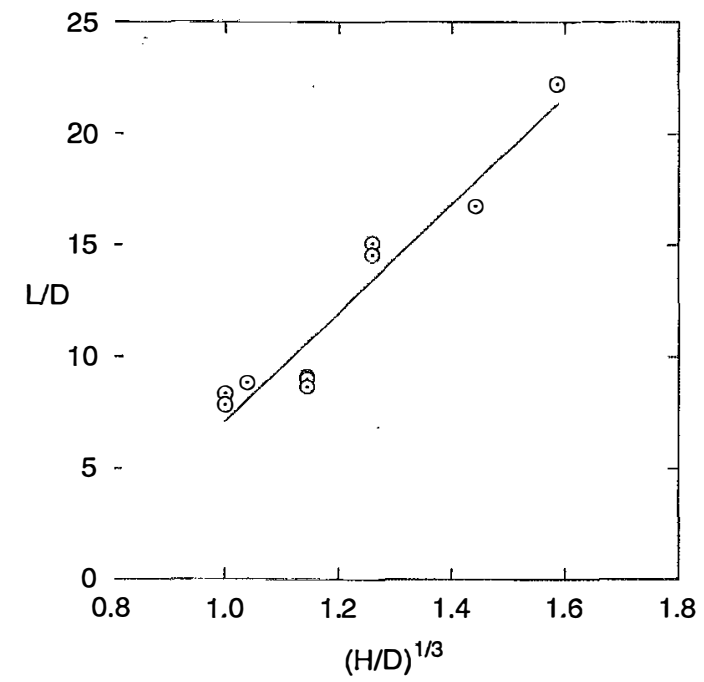

FIG. 6. Normalized reaction length as a function of $(H / D)^{1 / 3}$, at fixed stoichiometric ratio $\phi=\frac{1}{37}$. The solid line represents the least square line fitted to the data.

0.015 for the spreading rate; $\alpha$. The resulting value of 0.36 is peculiar, since one would expect a value greater than unity for $C$. This $C$ value implies that mixing occurs even faster than the entrainment of fresh reactants into the core. This is unrealistic and the issue will be discussed later.

Even though $\phi$ could not be varied extensively, runs were made with $\phi$ ranging from 0.016 to 0.20 . The larger mixture ratio runs were performed in the smaller $H / D$ settings in order to have the reaction length less than the maximum allowable depth. The limited range and small values of $\phi$ less than unity in our experiments barred us from plotting $L / D$ against $\phi$ for constant $H / D$. The normalized reaction length as a function of composite parameter $(1+\phi)^{1 / 3}(H / D)^{1 / 3}$ is shown in Fig. 7. The data from the previous graph are included in Fig. 7. The scatter is believed to be a manifestation of virtual origin variations when the gen-

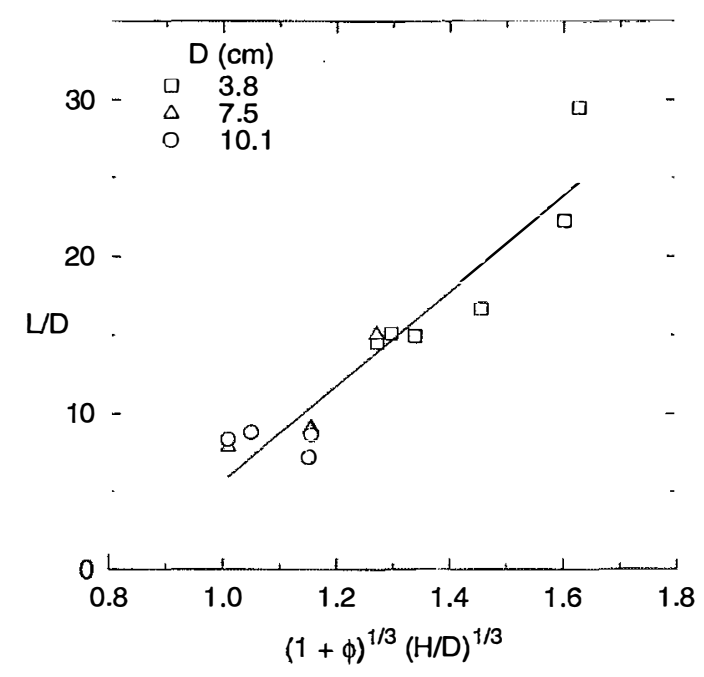

FIG. 7. Dependence of normalized reaction length on the composite parameter $(1+\phi)^{1 / 3}(H / D)^{1 / 3}$. The least square line fitted to the data is depicted by the solid line. 
erator geometry is altered. Despite the scatter, a least-squares line was fitted to the data. Again, assuming a mean spreading rate of 0.015 , the resulting value for proportionality constant C in Eq. (6) is calculated to be 0.45 . Although this value is greater than the one found from Fig. 6, it is still less than unity and impossible from the model's perspective.

An explanation for $C$ being less than unity is that the model does not take into account the detrainment of core material into the wake. Previous experiments have shown that the wake of a turbulent vortex ring contains vorticity as well as material ejected from the core.$^{8,9,11}$ The gross entrainment and detrainment rates of the vortex core have to remain in balance for self-similar turbulent rings, otherwise the ring will shrink or grow very rapidly. The small difference between the gross rates results in the relatively small net entrainment of the core. ${ }^{9}$

Prior to discoloration, a chemically reactive vortex core will leave behind parcels that are not mixed to the stoichiometric ratio. These parcels will be neutralized subsequently by the rapid mixing in the wake. The model assumes that every core parcel is retained once the core is formed. Although the model's assumption is incorrect, the proportionality of the entrainment and detrainment rates in self-similar vortex rings justifies the model's general predictions with respect to the aspect ratio and $\phi$ dependence. This is further validated by the agreement of model's predictions with the measurements. In fact, if one assumes that every entrained ambient parcel is uniformly mixed with the core material immediately, the value of $C$ would still be less than unity due to the loss of un-neutralized core material to the wake. Therefore, detrainment of turbulent vortex rings cannot be ignored when the mixing rate of the core is considered.

The reaction length of the fluid surrounding the core was also recorded in a number of runs. The stoichiometric mixture ratios for these runs could be increased to a value of $\phi \sim 5$. Measurements indicate that the reaction length of the fluid in the bubble surrounding the ring does not depend on the generator aspect ratio. For the large cylinder with a Reynolds number of $8.8 \times 10^{4}$, the fluid in the bubble had mixed 160 and 240 times more than the core fluid at 7.5 and 10 diameters from the generator, respectively. These ratios may vary with the generator geometry since the core mixing depends on $H / D$ as well as the initial volume. These findings are not surprising since previous experiments have already shown that the bubble processes a large volume of fluid and leaves the mixed fluid in the wake.

To investigate the effects of turbulent flow at the generator exit on the formation and mixing of vortex rings, a series of experiments were conducted in which the exit plane of the generator cylinder was covered by a wire mesh with solidity of $35 \%$ and wire thickness of $0.28 \mathrm{~mm}$. According to the previous studies in the literature ${ }^{18-20}$ the modified apparatus should create turbulent puffs. A "puff" is a mass of turbulent fluid having linear momentum and vorticity distributed throughout the moving mass. ${ }^{20}$ The flow resulting from placing the mesh against the generator exit was a distorted vortex ring and not a puff. In no case was it possible with the present geometry to create a puff in which the released material did not end up in a ring stmeture. This is in contrast to the findings of the previous experiments with similar geometries. ${ }^{18,19}$ The distorted vortex eventually reorganized itself (5-10D downstream) into a turbulent vortex ring with a large cross section.

The reaction length of the rings generated when the wire mesh was in place, was compared with the ordinary vortex rings. For the case of $D=3.8 \mathrm{~cm}$ and $H / D=4$, the reaction length was shorter by approximately $30 \%$ at comparable values of $\phi$. The reduction in the reaction length is speculated to be caused primarily by the enhanced initial mixing of the distorted ring. It is expected that for large values of $\phi$, the role of initial dilution and mixing would be reduced and the reaction length of distorted rings would get closer to the undisturbed ones.

\section{v. CONCLUSIONS}

Mixing in turbulent vortex rings was investigated by utilizing an aqueours acid-base chemical reaction. Visual observations revealed that the fluid in the bubble surrounding the vortex core gets mixed quite rapidly, near the vortex generator. On the other hand, the core mixed very slowly. Surprisingly, the mixing of core fluid was not azimuthally symmetric in a number of runs. A volumetric model for mixing of turbulent vortex rings was developed in which the reaction length, normalized by the cylinder diameter, is dependent upon the cylinder aspect ratio, the stoichiometric mixture ratio, the spreading rate and virtual origin of the ring. Although there is a Reynolds number effect at the lower $\operatorname{Re}_{0}$, the reaction length in the majority of runs appears to be Reynolds number independent, to within our measurement uncertainty. At a given stoichiometric mixture ratio, the reaction length becomes larger with increasing generator aspect ratio. Furthermore, detrainment of un-neutralized core material causes the measured reaction length to be less than that predicted by the model. These finding may be important in applications where mixing of vortex rings are considered.

\section{ACKNOWLEDGMENTS}

The help of Brett Taft during the experiments is gratefully acknowledged. The reviewers made valuable comments that improved the paper. The project was supported, in part, by the Research Development Council at Worcester Polytechnic Institute.

${ }^{1}$ J. S. Tumer, "Intermittent release of smoke from chimneys," Mech. Eng. Sci. 2, 97 (1960).

${ }^{2}$ A. Eroglu and R. E. Breidenthal, "Effects of periodic disturbances on structure and flame length of a jet in a crossflow," AIAA Paper No. 910317, 1991.

${ }^{3}$ T. Maxworthy, "The structure and stability of vortex rings," J. Fluid Mech. 51, 15 (1972).

${ }^{4} J$. P. Sullivan, S. E. Widnall, and S. Ezekiel, "Study of vortex rings using a laser Doppler velocimeter," AIAA J. 11, 1384 (1973).

${ }^{5} \mathrm{~N}$. Didden, "On the formation of vortex rings: Rolling-up and production of circulation," J. Appl. Math. Phys. 30, 101 (1979).

${ }^{6} \mathrm{~A}$. Glezer, "The formation of vortex rings," Phys. Fluids A 31, 3532 (1988).

${ }^{7}$ K. Shariff and A. Leonard, "Vortex rings," Annu. Rev. Fluid Mech. 24, 235 (1992).

${ }^{8}$ T. Maxworthy, "Turbulent vortex rings," J. Fluid Mech. 64, 227 (1974).

${ }^{9}$ A. Glezer and D. Coles, "An experimental study of a turbulent vortex ring," J. Fluid Mech. 211, 243 (1990). 
${ }^{10} \mathrm{G}$. M. Johnson, "An empirical model on the motion of turbulent vortex rings," AIAA J. 9, 763 (1971).

"T. Maxworthy, "Some experimental studies of vortex rings," J. Fluid Mech. 81, 465 (1977).

${ }^{12}$ D. W. Sallet and R. S. Widmayer, "An experimental investigation of laminar and turbulent vortex rings in air," Z. Flugwiss. 22, 207 (1974).

${ }^{13}$ D. Auerbach, "Stirring properties of vortex rings," Phys. Fluids A 3, 1351 (1991).

${ }^{14}$ K. B. Southerland, J. R. Porter Wi, W. J. A. Dahm, and K. A. Buch, "An experimental study of the molecular scale mixing process in an axisymmetric laminar vortex ring," Phys. Fluids A 3, 1385 (1991).

${ }^{15}$ D. S. Weddell, "Turbulent mixing in gas flames," Sc.D. thesis, Massachusetts Institute of Technology, 1941.
${ }^{16}$ W. J. A. Dahm and P. E. Dimotakis, "Measurements of entrainment and mixing in turbulent jets," AIAA J. 25, 1216 (1987).

${ }^{17} \mathrm{~J}$. E. Broadwell and R. E. Breidenthal, "A simple model of mixing and chemical reaction in a turbulent shear layer," J. Fluid Mech. 125, 397 (1982).

${ }^{18}$ H. R. Grigg and R. W. Stewart, "Turbulent diffusion in a stratified fluid," J. Fluid Mech. 15, 174 (1963).

${ }^{19} \mathrm{~J}$. M. Richards, "Puff motions in unstratified surroundings," J. Fluid Mech. 21, 97 (1965).

${ }^{20}$ L. S. G. Kovaznay, H. Fujita, and R. L. Lee, "Unsteady turbulent puffs," Adv. Geophys. 18b, 253 (1974). 